\section{Six-Minute Walk Test cut-off value identifying COPD patients with physical disability: a pilot study}

\section{Tho the Editors:}

\section{Sirs,}

Exercise limitation caused by dyspnea is a pivotal ifactor in the functional impairment of patients with chronic obstructive pulmonary disease (COPD) [1]. This means that it is essential to include exercise training programmes in the treatment of the disease, and particularly in pulmonary rehabilitation [2]. Among the tools used to measure exercise limitation, the 6-minute walk test (6MWT) has been recommended for a number of years, is widely used and may be performed using a standardised technique [3]. The 6MWT is also able to predict the resource utilisation [4] and the risk of death in patients with severe pulmonary impairment $[5,6]$. Notable differences have been reported according to the pathology. In fact, a cut-off value of a walking distance of 350 meters has been reported for COPD patients, compared with 207 metres for patients with idiopathic pulmonary fibrosis [7].

To the best of our knowledge there are no studies that have investigated the walking distance cutoff considering that the condition presents physical disability. The Barthel's index was introduced in 1965 to measure performance in activities of daily living, and can be used to estimate physical disability [8]. The aim of our study was to determine the walk distance cut-off in COPD patients with or without physical disability. Cut-off distances were assessed using the statistical method of Receiver Operating Characteristic (ROC) curves.

We progressively enrolled 100 patients with COPD referring to the outpatient setting of our Pulmonary Rehabilitation Unit. Subjects with previous courses of pulmonary rehabilitation and patients with other pulmonary diseases, such as fibrosis or pulmonary hypertension, were excluded. Each patient was administered a modified version of Barthel index, that consists of 10 items, with a score of 0-10 points for every variable [9]. The score ranges from 0 (complete disability) to 100 (no disability). According to the scores, 2 groups were formed: group A, patients with physical disability (Barthel score <100), and group B, patients without physical disability (Barthel score $=100$ ). All patients underwent the 6MWT. The walk distance and the percentage of predicted values in the 2 groups were analysed and compared by the Student $t$ test for unpaired data, setting the significance at $p<0.05$, and using the method of the ROC curves to identify the cut-off. The percentage of predicted values was calculated using the updated reference standards for healthy subjects [10]. For ROC curve analysis we used the computer program Labroc-1 (by C. Metz et al. University of Chicago,
U.S.), a modified version of the programme Rscore II, that establishes from the continuously distributed input data several operating points corresponding to a series of discriminator positions [11]. The cut-off was established by plotting the values of sensitivity (true positive results in patients with a Barthel score lower than 100) and 1-specificity ( 1 - false positive results in patients with a Barthel score equal to 100) obtained for each discriminator position and then determining the distance of the discriminator position from the ideal point represented by a sensitivity of 1 and a specificity of 1 , that is, a 1-specificity of 0 . In other words, each discrete point on the graph, called an operating point, is generated by using different cut-off levels for a positive test result. The optimal cut-off was obtained at the point of the curve at the minimal distance from the ideal point.

Group A (physical disability group) included 35 males and 15 females, mean age 71.7 years, (range 55-85 years); $\mathrm{FEV}_{1}$ mean value was $57.8 \%$ of predicted value. Group B (no physical disability group) included 33 males and 17 females, mean age 72.1 years (range 56-88 years); $\mathrm{FEV}_{1}$ mean value was $58.1 \%$ of predicted value. The frequency of patients with comorbidities was comparable in the 2 groups (76\% in group A, $72 \%$ in group B). Barthel index scored 76.4 \pm 18.2 in Group A vs. 100 in Group B, (the number required to be considered not to have a physical disability and therefore placed in the latter group). The mean distance walked during the 6MWT was $202.4 \pm 71.1$ metres in Group A compared to $418.6 \pm 72.1$ meters in Group B $(p<0.0001)$. The mean predicted value was $39.5 \pm 0.1 \%$ in Group A compared to $78.7 \pm 0.1 \%$ in Group B ( $p<$ 0.0001 ). Figure $1 \mathrm{~A}$ and $\mathrm{B}$ shows the ROC curves obtained by the distance walked (metres) and the percentage of predicted values in the 2 groups. The optimal cut-offs corresponded to 287 meters and to $59.6 \%$, respectively. The cut-off for walked distance had a sensitivity of $92.8 \%$, a specificity of $94.2 \%$, a positive predictive value of $93.8 \%$ and negative predictive value of $92 \%$. The cut-off for the percentage of predicted valued had a sensitivity of $93 \%$, a specificity of $93 \%$, a positive predictive value of $94 \%$ and negative predictive value of $94 \%$.

Among the negative outcomes of COPD, limitation in exercise capacity, that is mainly caused by dyspnea but also by systemic effects of the disease, reflects at best the functional deterioration. The $6 \mathrm{MWT}$ is a simple and valid tool to assess the functional status in COPD patients [3]. 6MWT reference standards for adults - male and female were recently assessed by analysing the distance walked by healthy subjects aged $40-80$ years during the test [10]. A study on a large population of COPD patients found that a distance of 350 meters was predictive of mortality, as assessed at followup of $55 \pm 30$ months [12]. However, there are no reported values of the 6MWT identifying physical disability in these patients. In the present pilot study, we used the ROC curve analysis to detect the cut-off distance walked during the 6MWT and the percentage of predicted values (according to the current reference standards) discriminating 


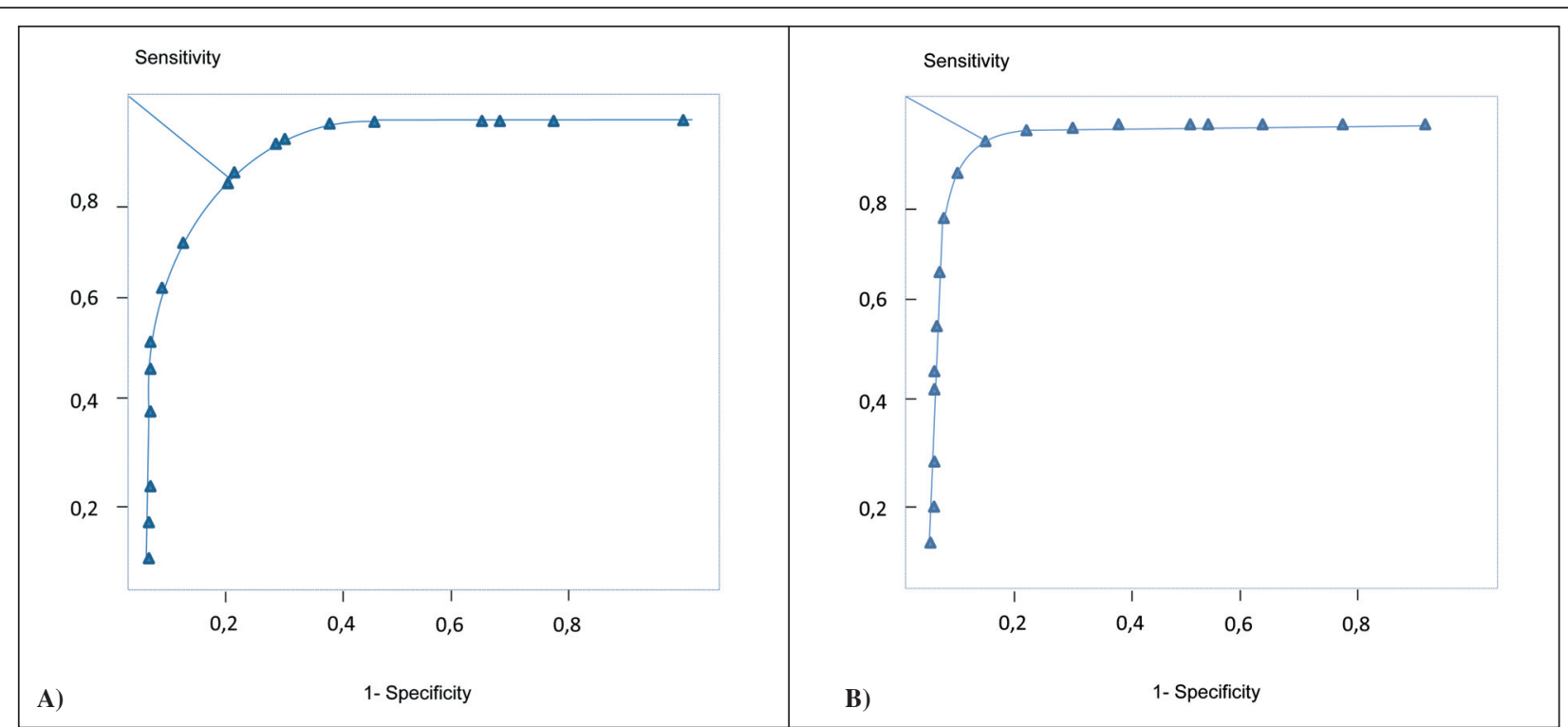

Fig. 1. - ROC curve identifying the cut-off of distance walked during a 6-minute walk test (1A) and \% of predicted value (1B) between two equal groups of patients with and without physical disability assessed by the Barthel index.

COPD patients with physical disability. Highly significant differences were found regarding both meters walked and percentage of predicted value $(p<0.0001)$ between patients with and without physical disability. By means of the ROC curve analysis, COPD patients with physical disability were identified using 287 meters as the cut-off walked distance and $59.5 \%$ as the cut-off of predicted value. Both cut-off had sensibility, specificity, positive predictive value and negative predicted value higher than $90 \%$.

These preliminary data indicate a possible utility of cut-off values of the 6MWT suggesting physical disability when evaluating COPD patients undergoing pulmonary rehabilitation. Studies on larger populations (based on a formal sample size calculation) with different grade of disease and disability need to be performed to assess reliable cut-off for such considerable parameter.

\section{Cristoforo Incorvaia1, Fulvia Paterniti1, Laura Pessina ${ }^{1}$, Roberta Caligiuri ${ }^{1}$, Franco Frati ${ }^{2}$, Mona-Rita Yacoub ${ }^{3}$, Gian Galeazzo Riario Sforza1}

1 Pulmonary Rehabilitation, ICP Hospital, Milan, Italy.

2 Institute of Pediatrics, University Department of Medical and Surgical Sciences, Perugia, Italy.

3 Allergy and Immunology Department, IRCCS San Raffaele Hospital, Milan, Italy.

Correspondence: Dr. Gian Galeazzo Riario Sforza, Via Bignami 1, 20126 Milan, Italy; e-mail: griariosforza@icp.mi.it

\section{References}

1. O'Donnell DE. Hyperinflation, dyspnea, and exercise intolerance in chronic obstructive pulmonary disease. Proc Am Thorac Soc 2006; 3: 180-4.

2. Troosters T, Casaburi R, Gosselink R, Decramer M. Pulmonary rehabilitation in chronic obstructive pulmonary disease. Am J Respir Crit Care Med 2005; 172: 19-38.

3. American Thoracic Society Statement. Guidelines for the six-minute walk test. Am J Respir Crit Care Med 2002; 166: 111-17.

4. Redelmeier DA, Bayoumi AM, Goldstein RS, Guyatt $\mathrm{GH}$. Interpreting small differences in functional status: the six minute walk test in chronic lung disease patients. Am J Respir Crit Care Med 1997; 155: 1278-82.

5. Gerardi DA, Lovett L, Benoit-Connors ML, et al. Variables related to increased mortality following outpatient pulmonary rehabilitation. Eur Respir J 1996; 9: 431-5.

6. Miyamoto S, Nagaya N, Satoh T, et al. Clinical correlates and prognostic significance of six-minute walk test in patients with primary pulmonary hypertension: comparison with cardiopulmonary exercise testing. $\mathrm{Am}$ J Respir Crit Care Med 2000; 161: 487-92.

7. Riario-Sforza GG, Incorvaia C. Mortality predictive capacity of the 6-min walk distance. Eur Respir J 2008; 32: 1132-33.

8. Mahoney FI, Barthel DW. Functional evaluation: the Barthel index. Md State Med J 1965; 14: 61-65.

9. Granger CV, Dewis LS, Peters NC, et al. Stroke rehabilitation: analysis of repeated Barthel index measures. Arch Phys Med Rehabil 1979; 60: 14-17.

10. Casanova C, Celli BR, Barria P, et al. The 6 minute walk distance in healthy subjects: reference standards from seven countries. Eur Respir J 2010. [Epub ahead of print]

11. Swets JA. Pickett RM. Evaluation of diagnostic systems: methods from signal detection theory. New York: Academic Press, 1982.

12. Cote CG, Casanova C, Marin JM, et al. Validation and comparison of reference equations for the 6-min walk distance test. Eur Respir J 2008; 31: 571-78. 